\title{
EL DISTANCIAMIENTO ENTRE LA TEORÍA Y LA PRÁCTICA EN LA LABOR DOCENTE, UN RETO PARA LAS ESCUELAS DE PROFESORADO
}

\author{
Erandi Atondo Rodríguez* \\ Universidad Autónoma de Sinaloa
}

\section{RESUMEN}

En este artículo se reflexiona sobre el distanciamiento existente entre la teoría y la práctica en la labor docente, así como sobre la importancia de las escuelas de profesorado para reducir dicha brecha, ya que es durante su paso por la formación inicial cuando los docentes pueden construir las herramientas cognitivas necesarias para anclar la teoría con la práctica y utilizar el vínculo resultante para el óptimo desempeño profesional. Se inicia este documento con una exposición puntual sobre el conocimiento de los profesores, la manera de acceder a éste, su naturaleza y los saberes que lo componen, enfatizando en el saber distintivo de la labor docente: el pedagógico. Se finaliza con una exposición sobre cómo debe ser el profesional práctico en la educación.

Palabras Clave: formación inicial docente, conocimiento práctico docente, escuelas de profesorado.

\section{THE DETACH BETWEEN THEORY AND PRACTICE IN TEACHING, A CHALLENGE FOR TEACHERS' SCHOOLS}

\section{Abstract}

This paper is a reflection about the distance between the theory and the practice in the teaching work, as well as on the importance of the teachers' schools to reduce this gap, since it is during its passage through the training initial that teachers can build the cognitive tools necessary to anchor the theory with practice and use the resulting link for optimal professional performance. The article begins with a specific presentation on the knowledge of teachers, the way to access it, its nature and the knowledge that compose it, emphasizing the distinctive knowledge of teaching: the pedagogical. It ends with a presentation on how the practical professional in education should be.

KEYwORDs: initial teacher education, teachers' practical knowledge, teacher schools. 


\section{INTRODUCCIÓN}

A partir de la década de los 90, la investigación educativa ha centrado su atención tanto en definir cuáles son los saberes que el profesor posee para desempeñar su práctica profesional como en establecer la naturaleza de los mismos; entendiendo que la conjunción de todos estos saberes constituye el conocimiento que el profesor utiliza para desempeñar su acción docente y que éste se construye sobre la práctica profesional.

En relación con lo que es el conocimiento de los profesores y la manera de acceder a él existe una nutrida discusión. Según Clandinin y Conelly (1987) el conocimiento es un «cuerpo de convicciones o significados conscientes o inconscientes que han surgido de la experiencia... y que se expresan en las acciones de una persona» (p. 59). Para Elbaz (1981) el conocimiento que poseen los profesores es complejo, dinámico y multifacético, lo han adquirido en la práctica y es usado para dar forma a la misma. Acceder a este conocimiento representa una situación problemática (Calderhead, 1988) en parte por el carácter complejo del mismo, y en parte porque las personas difícilmente quieren decir lo que dicen o dicen lo que quieren decir (Elbaz, 1981). Aquí vale mencionar lo que se conoce como teorías expuestas, que se refieren a lo que las personas manifiestan en su discurso, y teorías en uso, que son las que dan forma al comportamiento de las personas, es decir, que lo que se hace no se corresponde necesariamente con lo que se dice (Argyris y Schön, 1974). Para Badia y Monereo (2004), las posturas sobre el acceso al conocimiento del profesor se ubican en dos perspectivas psicológicas:

Por un lado, algunas perspectivas psicológicas consideran que dicho conocimiento se compone de representaciones esquemáticas que son fácilmente accesibles y explicitables por parte del profesor y, por lo tanto, se admite que una declaración articulada del contenido de dicho conocimiento supone una manifestación irrefutable de su saber profesional. Por el contrario, otras posiciones psicológicas mantienen que el conocimiento del profesor, en la mayoría de las ocasiones, no puede reducirse a aquello que es capaz de declarar y, por lo tanto, sostienen la existencia de un conocimiento de naturaleza implícita que únicamente se evidencia cuando es activado en el desarrollo de su actividad docente en el aula (p. 3).

La naturaleza del saber docente es otro aspecto que ha sido objeto de polémica, ya que las opiniones al respecto se polarizan entre la postura del enfoque cognitivo, que defiende que el conocimiento del profesor es una construcción personal

* E-mails: erandi.atondo.fce@uas.edu.mx, e.atondo.r@gmail.com. Es maestra en Educación por la Facultad de Educación de la Universidad Autónoma de Sinaloa y actualmente estudiante del Doctorado en Educación por la misma institución. Sus intereses profesionales se enfocan en la docencia universitaria e investigación, la formación profesional docente y el conocimiento práctico docente en profesores en formación inicial. 
elaborada con la maquinaria intelectual de cada docente, y la postura que defiende que el conocimiento es una construcción con base social y por tanto es un saber dado.

Para Clandinin y Conelly (1987) el conocimiento de los profesores tiene carácter personal, lo que no significa que pueda considerarse propiedad privada ni que esté socialmente abstraído; es, pues, un conocimiento personal mas no individual. Este conocimiento se construye a partir de la experiencia; pero cada construcción será particular ya que ésta depende de las prácticas reflexivas de cada sujeto. $\mathrm{Al}$ respecto, Tardif (2004) hace una mediación exponiendo que el saber de los educadores ni se construye individualmente ni está externamente dado, es decir: «Ni mentalismo, ni sociologismo». Para el autor, los profesores no construyen su saber en abstracto; pero tampoco es un saber totalmente dependiente de las fuerzas y mecanismos sociales; es, pues, «un interfaz entre lo individual y lo social, entre el actor y el sistema» (Tardif, 2004, p. 13).

Con base en lo anterior, es posible decir que los profesores construyen personalmente el conocimiento que utilizan para llevar a cabo su labor. Comienzan a hacerlo en la formación inicial y continúan a lo largo de su trayectoria profesional con la participación activa de su aparato cognitivo, influidos por los contextos, actores y relaciones que en el camino van encontrando:

El saber del profesorado no es un "foro íntimo" poblado de representaciones mentales, sino un saber siempre ligado a una situación de trabajo con otros (alumnos, colegas, padres, etc.), un saber anclado en una tarea compleja (enseñar), situado en un espacio de trabajo (aula, escuela), enraizado en una institución y en una sociedad (Tardif, 2004, p. 13).

Sobre cómo surge el conocimiento del profesor, existe un extenso debate, del cual se pueden rescatar los puntos de vista de algunos autores. Elbaz (1981) desarrolla su tesis sobre el conocimiento práctico; Clandinin (1985) dice que el conocimiento del profesor es experiencial, práctico, personal, teórico y cultural; Villar (1990) expone que el conocimiento no es un fenómeno estático, sino que se concibe en términos de construcción progresiva y que la fuente del conocimiento profesional es la práctica; para Estebaranz y Mingorance (1992) es un conocimiento práctico, personal, de origen grupal y con carácter contextual; para Marrero (1992) tiene carácter experiencial, asumiéndolo como la representación personal que, sobre sus experiencias culturales y sociales, construye cada docente; finalmente, Porlán y Rivero (1998) señalan que es un conocimiento experiencial-racional dividido en dos dimensiones: explícita y tácita. De las diversas posturas, se puede rescatar el factor común de que dicho conocimiento es personal, complejo, heterogéneo, está compuesto por diversos saberes y se construye sobre la experiencia de la práctica. En lo referente al contenido del conocimiento de los profesores, se puede decir que es de común acuerdo que éste se conforma por una articulación de distintos saberes. 


\section{LOS SABERES QUE COMPONEN EL CONOCIMIENTO DEL PROFESOR}

La investigación educativa, en su evolución, ha llegado a reconocer que el profesor conjuga un cúmulo de saberes para llevar a cabo su práctica profesional, empresa para la cual la obra de Lee Shulman (1987) ha servido de parteaguas y base para los distintos planteamientos que al respecto se pueden encontrar en la literatura especializada (Escudero, González y Rodríguez, 2018).

En Knowledge and teaching: foundations of the new reform, Shulman (1987) expone que los docentes poseen un conocimiento base para despeñar su labor profesional y que éste se compone de siete categorías: 1) el conocimiento del contenido, 2) el conocimiento didáctico general, 3) el conocimiento del currículo, 4) el conocimiento didáctico del contenido, 5) el conocimiento de los alumnos y de sus características, 6) el conocimiento de los contextos educativos y 7) el conocimiento de los objetivos, las finalidades y los valores educativos, y de sus fundamentos filosóficos e históricos.

De los componentes del saber docente, destaca la importancia del conocimiento didáctico del contenido como el distintivo para la enseńanza, es decir, es el elemento que diferencia la mente de un profesional de una disciplina específica y la del docente; por ejemplo, la diferencia entre la mente de un físico-matemático y la de un profesor de matemáticas. Según el autor, los conocimientos numerados anteriormente provienen de por lo menos cuatro fuentes:

1) la formación académica en la disciplina a enseñar; 2) los materiales y el contexto del proceso educativo institucionalizado (por ejemplo los currículos, los libros de texto, la organización escolar y la financiación, y la estructura de la profesión docente); 3) la investigación sobre la escolarización; las organizaciones sociales; el aprendizaje humano, la enseñanza y el desarrollo, y los demás fenómenos socioculturales que influyen en el quehacer de los profesores; y 4) la sabiduría que otorga la práctica misma (Shulman, 1987, p. 8).

Otro planteamiento importante en la obra de Shulman (1987) es el modelo de acción y razonamiento pedagógico, a partir del cual se explica que el profesor conjuga de una manera única los saberes contenidos en su conocimiento base para desempeñar la actividad docente; este razonamiento es lo que conduce y explica las acciones pedagógicas. El modelo se describe como un proceso de seis fases. El docente inicia la labor de enseńanza desde la comprensión crítica del contenido disciplinar. En seguida, esa comprensión será transformada con la finalidad de superar el plano personal y construir una imagen comprensible para los estudiantes; lograr lo anterior involucra las actividades cognitivas de preparación, selección y adaptación de contenidos. Realizado esto, pasa a la fase de enseńanza en la que se lleva a cabo el desempeño de la acción docente. De manera paralela el docente se ubica en la fase de evaluación, en la que verifica el correcto aprendizaje y/o detecta las malas interpretaciones por parte de los estudiantes. Ex post facto, el profesor reflexiona sobre la actividad docente desempeñada, lo que genera que él mismo aprenda de su expe- 
riencia. Finalmente, el proceso se cierra con una nueva comprensión más compleja de los estudiantes y de los procesos didácticos.

Otros autores han hablado sobre los saberes contenidos en el conocimiento del profesor. Elbaz (1981), en su tesis sobre el conocimiento práctico, expone que, aunque posiblemente inarticulado, el conocimiento de los profesores se compone de una gama de saberes que le guían en su práctica docente, éstos son: el conocimiento de la materia, el conocimiento de la organización del aula y las técnicas de enseñanza, el conocimiento de la estructura de las experiencias de aprendizaje y el contenido del currículum, el conocimiento de las necesidades, habilidades e intereses de los estudiantes, el conocimiento del marco de referencia de la escuela y de las comunidades aledañas y finalmente enuncia el conocimiento que posee el profesor de sus propias fortalezas y debilidades. Dicho conocimiento se articula de distintas formas según las tareas y problemas con que el profesor se enfrente en su ejercicio profesional y es lo que denomina «conocimiento práctico».

En su trabajo, Elbaz (1981) sintetiza el contenido del conocimiento práctico en cinco categorías: 1) de asignatura, 2) de currículum, 3) de instrucción, 4) de sí mismo y 5) del medio didáctico. Identifica también que este conocimiento se utiliza con base en cinco orientaciones: 1) situacional, 2) teórica, 3) personal, 4) social y 5) experiencial. Finalmente, con el propósito de encontrar la estructura necesaria para un fenómeno con la complejidad que se atribuye al que aquí se describe, la autora propone tres términos que reflejan los propósitos con que el profesor utiliza los conocimientos: 1) reglas de práctica, 2) principios de práctica y 3) imágenes (p. 49).

Otro desarrollo es el de Porlán y Rivero (1998), para quienes el conocimiento de los profesores es un conocimiento práctico que «no puede reducirse a conocimientos académicos, experienciales ni filosóficos» (p. 66); pero reconocen que éstos representan las fuentes y componentes de dicho conocimiento, de modo que explican que éste se constituye por los saberes metadisciplinares, que se refieren a la parte filosófica del conocimiento del profesor, son saberes generales y relevantes que consideran las cosmovisiones ideológicas, las perspectivas epistemológicas y las ontológicas. También están los saberes disciplinares, que se dividen en los de contenido, los de orden pedagógico (de la enseñanza y del aprendizaje) y los del estudio de los sistemas educativos. Finalmente, los autores reconocen a la experiencia profesional como fuente y componente del conocimiento del profesor, ésta se integra por los saberes rutinarios, principios y creencias personales y saberes curriculares sistematizados.

Para Tardif (2004, p. 26), «el saber docente se compone, en realidad, de diversos saberes provenientes de diversas fuentes». Éstos son: 1) los saberes profesionales, que son los pedagógicos y los producidos por las ciencias de la educación, son adquiridos en la formación inicial docente, 2) los saberes disciplinarios, son el conocimiento social correspondiente a diferentes campos del conocimiento, también se integran al conocimiento del docente mediante la formación (básica, inicial y continua), 3) los saberes curriculares, representan la organización mediante la cual las instituciones presentan los saberes sociales al estudiantado, toman la forma de programas escolares y, aunque los profesores tienen un acercamiento a ellos en la formación inicial, realmente son adquiridos en el ejercicio profesional por medio de 
la formación permanente; finalmente, 4) los saberes experienciales, que son aquéllos desarrollados por los profesores con base en su trabajo cotidiano y en el conocimiento de su medio, éstos toman la forma de rutinas y habilidades que se incorporan al conocimiento docente individual y colectivo (Tardif, 2004, pp. 26-31). Dicho lo anterior, se concluye que, para este autor, las fuentes de los saberes docentes son la formación básica, inicial docente y continua; la práctica profesional; y la socialización escolar.

Finalmente, Tamir (2005) considera que los profesores incorporan en su conocimiento 1) el saber de la materia y 2) el saber didáctico. El primero contempla los saberes disciplinarios; mientras que el segundo se divide en general y específico de la materia, considerando el conocimiento del estudiante, el conocimiento del currículo, las habilidades de explicación y las habilidades de evaluación.

De acuerdo con lo dicho anteriormente, es posible concluir que existe más o menos acuerdo sobre los saberes que componen el conocimiento del profesor. La diferencia radica en la clasificación y las relaciones que describe cada autor. Por ejemplo, en Shulman (1987) se percibe una clasificación más desglosada de los saberes, mientras que Porlán y Rivero (1998) manejan los mismos saberes, pero presentan una clasificación más condensada. A modo de síntesis, se puede decir que el conocimiento de los profesores se compone por saberes 1) disciplinares, 2) pedagógicos, 3) curriculares y 4) de la práctica; siendo el saber pedagógico el que distingue la práctica docente de otras profesiones.

\section{EL SABER PEDAGÓGICO: LA BASE DE LA PRÁCTICA PROFESIONAL DOCENTE Y LA IMPORTANCIA DE LAS ESCUELAS DE PROFESORADO PARA SU ADQUISICIÓN}

Todos los desarrollos teóricos sobre el conocimiento del profesor consideran en su clasificación de saberes uno particular de la profesión docente. Se reconoce que el profesor, además de dominar los contenidos de su disciplina, debe ser capaz de transmitirlos eficazmente a partir de conocimientos relativos a las ciencias de la educación y de habilidades pedagógicas (Tardif, 2004), para lo que se vale, además, de los conocimientos curriculares. Esos conocimientos de la ciencia de la educación y de la ideología pedagógica representan el distintivo del conocimiento del profesor y lo facultan para desarrollar una buena práctica docente.

Shulman (1987) hace referencia al conocimiento didáctico del contenido; Elbaz (1981) lo menciona como el conocimiento del medio didáctico; Porlán y Rivero (1998) lo consideran en el conocimiento disciplinar como saberes de la enseñanza y el aprendizaje, es decir, de didácticas específicas; Tardif (2004) hace referencia a los conocimientos profesionales; mientras que Tamir (2005) habla de conocimiento didáctico general y conocimiento didáctico específico. Para ahondar en el tema, en adelante se utilizará el término conocimiento pedagógico para hacer referencia a este conocimiento particular de la profesión docente.

Es claro que, para desarrollar una buena práctica profesional, el profesor no puede omitir ninguno de los saberes; pero es con base en el conocimiento pedagó- 
gico que el docente desarrolla la capacidad de desempeñar la labor de enseñanza, ya que a partir de él desarrolla una racionalidad que le permite resolver, en su práctica profesional, problemas basándose en la aplicación de teorías y técnicas científicas ya construidas (Schön, 2010).

Como se ha dicho anteriormente, el conocimiento pedagógico involucra los saberes teóricos de las ciencias de la educación y de la ideología pedagógica, y representa el lenguaje especializado y exclusivo de la profesión docente, éste es adquirido en las escuelas de profesorado y, en teoría, debería perfeccionarse durante toda la carrera profesional de la mano con los saberes experienciales que se van desarrollando.

Las escuelas de profesorado juegan un papel estratégico para que los docentes adquieran este saber, ya que, como expone Berliner (2000), la formación inicial docente tiene la responsabilidad de dotar a los futuros profesores del lenguaje profesional necesario para desempeñar su labor. Tardif (2004) añade que la función de la formación inicial del profesorado es proporcionar a los futuros docentes los conocimientos teóricos y técnicos que los preparen para el ámbito laboral. Sin embargo, el campo de estudio preocupado por los aspectos concernientes a la educación no es un campo estático, por lo que el docente debe seguir renovando sus conocimientos pedagógicos durante toda su carrera profesional mediante la formación continua.

De esta manera, se puede afirmar que es en la formación inicial cuando los docentes deben adquirir los conocimientos teóricos que consolidarán su saber pedagógico. Este saber pedagógico precede y, en teoría, debería dominar la práctica (Tardif, 2004), aunque al no provenir de ella misma, es muy probable que permanezca en el abandono, en parte debido al llamado choque con la realidad profesional que experimentan los profesores en sus años principiantes (Jacobo, 1997; Armenta, 2012) y, en parte, debido a la lejanía que existe en la formación inicial docente entre la teoría y la práctica, lo que provoca que los profesores no establezcan vínculo con el conocimiento pedagógico, facilitando su abandono en las primeras turbulencias de la labor docente. Así es que, en su práctica profesional madura, los profesores se refugian principalmente en el conocimiento que les brinda la práctica.

\section{EL CONOCIMIENTO PRÁCTICO DE LOS DOCENTES}

En los desarrollos teóricos, se menciona que el conocimiento del profesor se produce a partir de la práctica y que al mismo tiempo otorga forma a la práctica misma. Se menciona también que el conocimiento del profesor es de carácter situado y que su fuente es la experiencia.

Así, surge el planteamiento del conocimiento práctico, que defiende que lo que saben los profesores se construye a partir de las experiencias personales y profesionales, aunque debe ser fundamentado en las teorías formales, lo que no ocurre todo el tiempo, ya que, como se ha mencionado anteriormente, desde la misma formación inicial, la práctica y la teoría se encuentran tan distanciadas que parece ser una empresa sumamente complicada lograr que el estudiante de profesorado construya en su razonamiento procesos de anclaje entre teoría y práctica, siendo en la mayoría de los casos que se abandona la primera en el desempeńo profesional. 
Desde la perspectiva de las teorías implícitas se define que el conocimiento práctico «está impregnado de creencias, mejor o peor organizadas en sistemas que se forman desde edades tempranas» (Pérez-Gómez, 2010, p. 173), es decir, que es un conocimiento tácito, poco articulado, formado con base en experiencias personales y profesionales, con prioridad en los aspectos afectivos del individuo sin dejar mucho margen a los requerimientos teóricos.

Para responder al argumento anterior, Pérez-Gómez (2010) hace referencia al término teorización de la práctica (Hagger y Hazel, 2006), con el cual se dice que el docente, para mejorar su práctica profesional y/o abandonar vicios pedagógicos, debe reflexionar sobre su labor sirviéndose de los resultados de la investigación educativa más reciente. De esta manera, los procesos de reflexión e investigación permiten al docente mejorar su práctica educativa. Lo anterior hace visible que a partir de procesos de vinculación experiencia-teoría por medio de los ejercicios de reflexión, los profesores mejoran su propio conocimiento práctico. Dicho esto, se puede concluir que sólo a partir de los procesos de reflexión el docente tendrá la capacidad de abandonar el plano de lo inconsciente en su labor docente. Para lograr esto, debe convertirse en un profesional práctico reflexivo (Schön, 1998; 2010).

Para la comprensión de la idea del conocimiento profesional práctico, son necesarios tres conceptos desarrollados por Schön (1988) en El profesional reflexivo. Cómo piensan los profesionales cuando actúan. En primer lugar, el concepto de conocimiento en la acción, que se refiere a lo que las personas que hacen cosas conocen de las cosas que están haciendo. En segundo lugar, se presenta el concepto de reflexión en la acción, que habla de que las personas, con frecuencia, piensan sobre lo que hacen al mismo tiempo que actúan. Schön denomina a este componente del pensamiento práctico «reflexión en y durante la acción». Se puede profundizar en lo anterior diciendo que, sobre la racionalidad de primer orden que orienta la actividad práctica, se supone una racionalidad de segundo orden que está dialogando de manera implícita con la actuación del docente al tiempo que cuestiona y evalúa sus soluciones. En muchas ocasiones el profesor se cuestiona de manera inconsciente cómo solucionar de mejor manera las problemáticas, eso es el proceso de reflexión en la acción, que es un proceso vivo y muy dinámico; pero para poder hacerlo útil, es necesario mover estas reflexiones al terreno de lo explícito. Finalmente, con el concepto reflexión sobre la acción se considera el análisis que a posteriori realizan los profesionales sobre las características y procesos de su propia acción. Es la utilización del conocimiento para describir, analizar y evaluar las huellas que en la memoria corresponden a la intervención pasada.

El profesional práctico reflexivo (Schön, 1998; 2010) no se limita a la profesión docente, cualquier profesional puede serlo, siempre y cuando implique la reflexión en y sobre su acción. Lo que aquí nos interesa es la labor docente, y un profesional práctico docente es aquél que tiene la capacidad de resolver cualquier situación que se le presente en el aula. Este sujeto debe tener capacidad previsora, ya que la labor docente está de manera permanente en escenarios de problemas potenciales. El profesor entonces, como práctico, debe ser capaz de resolver cualquier problemática resultante sin importar las particularidades de las condiciones personales y del entorno, es decir, que un buen profesional reflexivo debe saber solucionar. 
En lo respectivo a la formación inicial de profesionales, en cuatro años no es posible considerar la cantidad de problemas que se pueden presentar en la práctica profesional, por lo que la preocupación debe ser dotar a los futuros profesionistas con las capacidades y habilidades que les puedan servir para hacer frente a su práctica.

El pensamiento del práctico reflexivo debería tener el conocimiento de la acción, la reflexión en la acción y la reflexión sobre la acción. El profesional que hace todo esto se convierte en un investigador, construye una teoría adecuada a la singular situación de su escenario y elabora en su interacción con la situación un diseńo flexible de enfoque progresivo que experimenta y reconduce de forma continua. En esta actuación reflexiva se asientan las bases de la evolución del pensamiento práctico en el desarrollo profesional. Cuando el profesional tiene la capacidad de la investigación será más hábil para resolver las situaciones problemáticas.

Al respecto, Korthagen (1993) dice que los procesos de reflexión guían a una conclusión que posiciona al profesional en un punto de partida nuevo que le permite avanzar. Es por esto por lo que hay que revisar lo que ha pasado, lo que implica tomar conciencia de ciertas situaciones, permitiendo la creación de alternativas que posicionaran al docente en un lugar diferente al estadio inicial. El conocimiento generado por este medio aporta nuevas herramientas metodológicas y mentales para el mejoramiento de la profesión del docente. La situación es que gran cantidad de profesionales resuelven acciones de manera implícita pero no tienen la habilidad de reflexión sobre esa acción; por lo tanto, la resolución del problema no tiene mayor trascendencia. Es muy peligroso no reflexionar sobre lo que se hace, ya que se pueden otorgar facultades sanadoras a herramientas meramente paliativas por haber quedado en la primera impresión; al profesional que cae en este vicio no se le puede considerar un práctico reflexivo.

El conocimiento profesional práctico debe ser un ir y venir de la práctica a la teoría y de la teoría a la práctica, explicitando por medio de la reflexión a las teorías que nos han conducido en la resolución de las situaciones de conflicto, es por esto por lo que Argyris, Putnam y Smith (1985) afirman que la reflexión sobre la acción es un componente esencial del proceso de aprendizaje permanente que constituye la formación del profesional.

Si bien la práctica reflexiva posiciona al docente en una situación más adecuada para hacer frente a la labor educativa, no debería ser el único tipo de reflexión que se active en el desempeño profesional. Jacobo (2009) propone, en El profesionalismo integrado «diversificar sus objetos de reflexión en tres dimensiones: práctica, técnica y social» (p. 109), tríada recuperada de las aportaciones de Liston y Zeichner (1993). La dimensión práctica tiene como punto de partida las experiencias docentes, se ampara en el sentido común y revisa los procesos ejecutivos de la enseńanza, nos es posible vincular esta dimensión con las propuestas de Donald Schön (1988) expuestas anteriormente. La dimensión técnica es el intento por trascender del conocimiento experiencial. Por medio de ésta, se busca dar explicaciones teóricas a las problemáticas profesionales, planteamiento que tiene relación con la idea de teorizar la práctica (Hagger y Hazel, 2006) expuesta líneas arriba. Finalmente, la dimensión social implica que la labor del docente va más allá de lo académico y se debe esforzar también en formar sujetos capaces de llevar una óptima vida en sociedad. 
La práctica reflexiva que se ha descrito no necesariamente debe ser tridimensional, el profesor debe saber combinar las dimensiones de acuerdo a los contextos y las problemáticas que enfrente (Jacobo, 2009), por lo que resulta indiscutible que este ejercicio de reflexión integrada «tiene mayor demanda cognitiva» (p. 110), sobre todo al activar la reflexión social.

Debemos rescatar que para Jacobo (2009), el profesionalismo integrado «se adquiere de manera contextualizada» (p. 111), por lo que la formación inicial concebida casi exclusivamente en las aulas de las instituciones formadoras de docentes no es suficiente para dotar al futuro profesor de las habilidades requeridas, es necesario, pues, acercarla cada vez más a los espacios reales de trabajo.

No queda más que apuntar a la necesidad de reflexionar sobre si la formación docente atiende a las necesidades de la sociedad actual y concluir dejando algunas interrogantes con la ilusión de abonar y refrescar al debate educativo actual: ¿los docentes de la actualidad pueden ser considerados profesionales reflexivos?, ¿las escuelas de profesorado han contribuido para lograr esta empresa?, ¿cómo podemos imaginar las escuelas de profesorado para formar profesionales prácticos?, ¿permite el diseño de la formación inicial docente que los estudiantes realicen conexiones entre teoría y práctica?, de entre muchas otras inquietudes que se espera puedan surgir. 


\section{REFERENCIAS}

Argyris, C. y Schön, D. (1974). Theory in practice: Increasing profesional effectiveness. California, Estados Unidos de América: Jossey-Bass.

Argyris, C., Putman, R. y McLain, D. (1985). Action Science. Concepts, Methods, and Skills for Research and Intervention. San Francisco, Estados Unidos de América: Jossey-Bass Publishers.

Badia, A. y Monereo, C. (2004). «La construcción del conocimiento profesional docente. Análisis de un curso de formación sobre la enseñanza estratégica». Anuario de Psicología, 35(1), 47-70.

Berliner, D. (2000). «A personal response to those who bash teacher education». Journal of teacher education, 51(5), 358-371. Doi: 10.1177/0022487100051005004.

Calderhead, J. (1992). Teachers' Professional Learning. Londres, Inglaterra: The Falmer Press.

Clandinin, D. (1985). «Personal practical knowledge: a study of teachers' classroom images». Curriculum Inquiry, 15(4), 361-385.

Clandinin, D. y Connelly, F. (1987). «Teachers' personal Knowledge: What counts as 'personal' in studies of the personal». Journal of Curriculum Studies, 19(6), 487-500.

Elbaz, F. (1981). «The teacher's practical knowledge: Report of a case study». Curriculum Inquiry, 11(1), 43-71.

Escudero Muñoz, J.M., González González, M.T. y Rodríguez Entrena, M.J. (2018). «Los contenidos de la formación continuada del profesorado: ¿Qué docentes se están formando?». Educación XX1, Revista de la Facultad de Educación, 21(1), 157-180. UNED.

Estebaranz, A. y Mingorance, P. (1992). «Proceso de pensamiento en los profesores y desarrollo curricular», en Estebaranz, A. y Sánchez, V. (eds.). Pensamiento de profesores y desarrollo profesional. Volumen I: Conocimiento y teorías implícitas (pp. 375-388). Sevilla, España: Universidad de Sevilla, Secretariado de Publicaciones.

Hager, H. y Hazel, H. (2006). Learning teaching from teachers: Realissing the potencial of schoolbassed teacher education. Buckingham, Inglaterra: Open University Press.

Jacobo-García, H. (2009). El profesionalismo integrado. Un nuevo modo de ser educador. México: Plaza y Valdés.

Korthagen, F. (1993). «Two modes of reflection». Teacher \& Teacher education, 9(3), 317-326.

Korthagen, F. (2010). «La práctica, la teoría y la persona en la formación del profesorado». Revista Interuniversitaria de Formación de profesorado, 68, 83-101.

Liston, D. y Zeichner, K. (1993). Formación del profesorado y condiciones sociales de la escolarización. España: Morata.

Marrero, J. (1992). «Las teorías implícitas del profesorado: un puente entre la cultura y la práctica de la enseñanza», en Estebaranz, A. y Sánchez, V. (eds.). Pensamiento de profesores y desarrollo profesional. Volumen I: Conocimiento y teorías implícitas (pp. 9-22). Sevilla, España: Universidad de Sevilla, Secretariado de Publicaciones.

PÉrez-Gómez, Á. (2010). «La naturaleza del conocimiento práctico y sus implicaciones en la formación de docentes». Infancia y aprendizaje; journal for the study of educational development, 33(2), 171-177. Doi: 10.1174/021037010791114652.

Porlán, R. y Rivero, A. (1998). El conocimiento de los profesores. Sevilla, España: Díada. 
Schön, D. (1998). El profesional reflexivo. Cómo piensan los profesionales cuando actúan. Barcelona, España: Paidós.

SснӧN, D. (2010). La formación de los profesionales reflexivos: hacia un nuevo diseño de la enseñanza y el aprendizaje en las profesiones. Barcelona, España: Paidós.

Shulman, L. (1987). «Knowledge and Teaching: Foundations of the New Reform». Harvard Educational Review, 57(1), 1-21.

TAmir, P. (2005). «Conocimiento profesional y personal de los profesores y de los formadores de profesores». Profesorado. Revista de currículum y formación del profesorado, 9(2).

TARdif, M. (2004). Los saberes del docente y su desarrollo profesional. Madrid, España: Narcea.

VILlar, L. (1990). El profesor como profesional: formación y desarrollo personal. Granada, Espańa: Servicio de Publicaciones de la Universidad de Granada. 